\title{
On the Holomorphic Solution of Non-linear Totally Characteristic Equations
}

\author{
CHEN HUA and Hidetoshi TAHARA
}

\begin{abstract}
The paper deals with a non-linear singular partial differential equation: (E) $t \partial / \partial t=F(t, x, u, \partial u / \partial x)$ in the holomorphic category. When (E) is of Fuchsian type, the existence of the unique holomorphic solution was established by Gérard-Tahara [2]. In this paper, under the assumption that (E) is of totally characteristic type, the authors give a sufficient condition for (E) to have a unique holomorphic solution. The result is extended to higher order case.
\end{abstract}

Keywords. Nonlinear, singular partial differential equation, holomorphic solution.

Classification. Primary 35A07; Secondary 35A10, 35A20

\section{Introduction and Main Result.}

Let $(t, x) \in \mathbf{C}_{t} \times \mathbf{C}_{x}$, we consider the following non-linear first order singular partial differential equation

$$
t \frac{\partial u}{\partial t}=F\left(t, x, u, \frac{\partial u}{\partial x}\right)
$$

where $u=u(t, x)$ is an unknown function, $F(t, x, u, v)$ is a function with respect to the variables $(t, x, u, v) \in \mathbf{C}_{t} \times \mathbf{C}_{x} \times \mathbf{C}_{u} \times \mathbf{C}_{v}$. Further we assume the following conditions: 
(H1) $F(t, x, u, v)$ is a holomorphic function defined in a neighborhood of the origin $(0,0,0,0) \in \mathbf{C}_{t} \times \mathbf{C}_{x} \times \mathbf{C}_{u} \times \mathbf{C}_{v}$.

(H2) $F(0, x, 0,0) \equiv 0$ near $x=0$.

Thus the function $F(t, x, u, v)$ may be expressed in the form:

$$
F(t, x, u, v)=\alpha(x) t+\beta(x) u+\gamma(x) v+R_{2}(t, x, u, v),
$$

where

$$
\alpha(x)=\frac{\partial F}{\partial t}(0, x, 0,0), \quad \beta(x)=\frac{\partial F}{\partial u}(0, x, 0,0), \quad \gamma(x)=\frac{\partial F}{\partial v}(0, x, 0,0),
$$

and the degree of $R_{2}(t, x, u, v)$ with respect to $(t, u, v)$ is greater than or equal to 2.

If $\gamma(x) \equiv 0$ near $x=0$, then $\beta(x)$ is the indicial exponent of (1), and Gérard-Tahara [2] gives that, if $\beta(0) \notin\{1,2, \cdots\}$, the equation (1) has a unique holomorphic solution $u(t, x)$ near a neighborhood of $(0,0) \in \mathbf{C}_{t} \times \mathbf{C}_{x}$ with $u(0, x) \equiv 0$ near $x=0$. The condition " $\gamma(x) \equiv 0$ near $x=0$ " means that the linearlized equation of (1) is "Fuchsian type"; so, in this case, the equation (1) is called non-linear Fuchsian type partial differential equation (or is called "Briot-Bouquet type equation" in $[1,2]$ ).

Remark. Quite recently, Yamane [7] also studied the case for the nonlinear Fuchsian type PDE whose indicial exponent takes a positive integer value.

If $\gamma(x) \not \equiv 0$ and $\gamma(0) \neq 0$, then we can use the implicit function theorem to solve $v$ from the equation (1), then, by using Cauchu-Kowalewski Theorem, we can deduce easily that the equation (1) has a unique holomorphic solution $u(t, x)$ with $u(0, x) \equiv 0$ and $u(t, 0) \equiv 0$ near $(0,0) \in \mathbf{C}_{t} \times \mathbf{C}_{x}$.

So we are only interested in the case of $\gamma(x) \not \equiv 0$ and $\gamma(0)=0$, i.e. the case of $\gamma(x)=x^{p} c(x)$, where $p$ is a positive integer and $c(0) \neq 0$. In this case, the equation (1) is called totally characteristic type; and $\beta(x)+\gamma(x) \partial_{x}$ is the indicial operator of (1). We know that there is an essential difference between the case of $p=1$ and the case of $p \geq 2$, since, in the case of $p \geq 2$, the indicial operator $\beta(x)+\gamma(x) \partial_{x}$ has an irregular singularity at $x=0$.

In this paper, we shall consider the simplest case, i.e.

(H3) $\gamma(x)=x c(x)$, and $c(0) \neq 0$

and the case of $\gamma(x)=x^{p} c(x)$, for $p \geq 2$ will be studied in the forthcoming paper. 
We have the following result:

Theorem 1. Under the conditions (H1), (H2) and (H3), if $\operatorname{Re} c(0)<0$ and $\frac{\beta(0)-k}{c(0)} \notin \mathbf{Z}_{-}=\{0,-1,-2, \cdots\}$ for any $k \geq 1$, then the equation (1) has a unique holomorphic solution $u(t, x)$ near $(0,0) \in \mathbf{C}_{t} \times \mathbf{C}_{x}$ with $u(0, x) \equiv 0$ near $x=0$.

\section{Proof of Main Result.}

First we put formally

$$
u(t, x)=\sum_{k=1}^{\infty} u_{k}(x) t^{k}
$$

Then introduce the formal series (3) into the equation (1) and compare the coefficients of $t^{k}$ in two sides of the equation, we have

$$
\begin{aligned}
u_{1} & =\alpha(x)+\beta(x) u_{1}+\gamma(x) \frac{\partial u_{1}}{\partial x}, \\
2 u_{2} & =\beta(x) u_{2}+\gamma(x) \frac{\partial u_{2}}{\partial x}+f_{1}\left(u_{1}, \frac{\partial u_{1}}{\partial x}\right), \\
3 u_{3} & =\beta(x) u_{3}+\gamma(x) \frac{\partial u_{3}}{\partial x}+f_{2}\left(u_{1}, u_{2}, \frac{\partial u_{1}}{\partial x}, \frac{\partial u_{2}}{\partial x}\right),
\end{aligned}
$$

Thus we obtain the following recursive formula

$\gamma(x) \frac{\partial u_{k}}{\partial x}+(\beta(x)-k) u_{k}=f_{k-1}\left(u_{1}, \cdots, u_{k-1}, \frac{\partial u_{1}}{\partial x}, \cdots, \frac{\partial u_{k-1}}{\partial x}\right)$, for $k \in \mathbb{N}$,

where $\gamma(x)=x c(x)$, and $f_{0}(x)=-\alpha(x)$, so

$$
x \frac{\partial u_{k}}{\partial x}+\left(\frac{\beta(x)-k}{c(x)}\right) u_{k}=\frac{1}{c(x)} f_{k-1}=\tilde{f}_{k-1}, \text { for } k \in \mathbb{N} .
$$

Denoting $\frac{\beta(x)-k}{c(x)}=\lambda_{k}(x)$, we easily see that if $l_{k}(0) \notin \mathbf{Z}_{-}=$ $\{0,-1,-2, \cdots\}$, then for any $k \geq 1$, the equation (5) has a unique holomorphic solution $u_{k}(x)$ near $x=0$ and moreover we see that all $u_{k}(x)$ are holomorphic in a common neighborhood of $x=0$. It remains to prove that the formal series solution (3) is convergent near $(0,0) \in \mathbf{C}_{t} \times \mathbf{C}_{x}$.

We need the following two lemmas, which will be useful for the proof of the main result. 
Lemma 1. If $\operatorname{Re} c(0)<0$, then there exists $K>0$, large enough, such that

$$
\left\|u_{k}\right\|_{r} \leq \frac{C}{k}\left\|\tilde{f}_{k-1}\right\|_{r}, \quad \text { for } k \geq K \quad(\exists C>0),
$$

where $u_{k}$ is the solution of (5), and the norm $\|\cdot\|_{r}$ (for $r>0$ small enough) is defined by $\|f\|_{r}=\max _{|x| \leq r}|f(x)|$.

Proof. From the equation (5), we have

$$
\frac{d}{d x}\left[e^{\int_{a}^{x} \frac{\lambda_{k}(s)}{s} d s} u_{k}(x)\right]=e^{\int_{a}^{x} \frac{\lambda_{k}(s)}{s} d s} \tilde{f}_{k-1}(x) \frac{1}{x},
$$

where $a$ is a point in the segment $l(0, x)$ joining the two points 0 and $x$ in the complex plane. Thus

$$
\int_{a}^{x} \frac{d}{d y}\left[e^{\int_{a}^{y} \frac{\lambda_{k}(s)}{s} d s} u_{k}(y)\right] d y=\int_{a}^{x} e^{\int_{a}^{y} \frac{\lambda_{k}(s)}{s} d s} \tilde{f}_{k-1}(y) \frac{1}{y} d y
$$

that is

$$
e^{\int_{a}^{x} \frac{\lambda_{k}(s)}{s} d s} u_{k}(x)-u_{k}(a)=\int_{a}^{x} e^{\int_{a}^{y} \frac{\lambda_{k}(s)}{s} d s} \tilde{f}_{k-1}(y) \frac{1}{y} d y
$$

and therefore

$$
u_{k}(x)=e^{-\int_{a}^{x} \frac{\lambda_{k}(s)}{s} d s} u_{k}(a)+\int_{a}^{x} e^{-\int_{y}^{x} \frac{\lambda_{k}(s)}{s} d s} \tilde{f}_{k-1}(y) \frac{1}{y} d y .
$$

Now, letting $a \rightarrow 0$ in $l(0, x)$ we have

$$
e^{-\int_{a}^{x} \frac{\lambda_{k}(s)}{s} d s} u_{k}(a) \rightarrow 0,
$$

because, for $k$ large enough, we know $\operatorname{Re} \lambda_{k}(0)>0$.

Hence we have

$$
u_{k}(x)=\int_{0}^{x} e^{-\int_{y}^{x} \frac{\beta(s)-k}{s c(s)} d s} \tilde{f}_{k-1}(y) \frac{1}{y} d y
$$

this implies, for $r>0$ small enough, that

$$
\left\|u_{k}\right\|_{r} \leq \max _{|x| \leq r}\left|\int_{0}^{x} e^{-\int_{y}^{x} \frac{\beta(s)-k}{s c(s)} d s} \frac{1}{y} d y\right| \cdot\left\|\tilde{f}_{k-1}\right\|_{r} .
$$


Since $c(0) \neq 0$, then $\frac{\beta(x)}{c(x)}$ is bounded near $x=0$, thus there exists a constant $C_{1}$, such that

$$
\left|e^{-\int_{y}^{x} \frac{\beta(s)}{s c(s)} d s}\right| \leq e^{C_{1} \int_{|y|}^{|x|} \frac{1}{\sigma} d \sigma}=e^{C_{1} \ln \left(\frac{|x|}{|y|}\right)}=\left(\frac{|x|}{|y|}\right)^{C_{1}} .
$$

Next, we set $C_{2}=-\operatorname{Re} c(0)>0$, then near $x=0$ we have

$$
\left|e^{k \int_{y}^{x} \frac{1}{s c(s)} d s}\right| \approx e^{\frac{k}{\operatorname{Rec}(0)} \ln \left(\frac{|x|}{|y|}\right)}=\left(\frac{|x|}{|y|}\right)^{-\frac{k}{C_{2}}} .
$$

Thus we obtain, for a constant $c>0$, that

$$
\left|\int_{0}^{x} e^{-\int_{y}^{x} \frac{\beta(s)-k}{s c(s)} d s} \frac{1}{y} d y\right| \leq c \int_{0}^{|x|}\left(\frac{|x|}{\eta}\right)^{C_{1}-\frac{k}{C_{2}}} \frac{1}{\eta} d \eta=c|x|^{C_{1}-\frac{k}{C_{2}}} \int_{0}^{|x|} \eta^{\frac{k}{C_{2}}-C_{1}-1} d \eta .
$$

Observe $C_{2}>0$, so if we choose $K>0$ large enough, suth that $\frac{k}{C_{2}}-C_{1}-$ $1 \geq 0$ for any $k \geq K$, then

$$
\int_{0}^{|x|} \eta^{\frac{k}{C_{2}}-C_{1}-1} d \eta=\frac{C_{2}}{k-C_{1} C_{2}} \cdot|x|^{\frac{k}{C_{2}}-C_{1}} .
$$

Hence we can choose a constant $C>0$, suth that for any $k \geq K$ we have $\frac{c C_{2}}{k-C_{1} C_{2}} \leq \frac{C}{k}$, this implies, combining with (7) and (8), that Lemma 1 holds.

Lemma 2. Let $R>0$ and $f(x)$ be a holomorphic function on $D_{R}=\{x \in$ $\mathrm{C}|| x \mid \leq R\}$. If for any $r, 0<r<R, f(x)$ satisfies

$$
\max _{|x| \leq r}|f(x)| \leq \frac{c}{(R-r)^{a}},
$$

for some $c>0$ and $a \geq 0$, then we have

$$
\max _{|x| \leq r}\left|\frac{\partial f}{\partial x}(x)\right| \leq \frac{(a+1) e c}{(R-r)^{a+1}}, \text { for } 0<\forall r<R .
$$

The proof of Lemma 2 is well-known, cf. Lemma 5.1.3 of [6] (also see [5]).

Now let us prove Theorem 1. First we expand the remainder term $R_{2}(t, x, u, v)$ of (2) into Taylor series with respect to $(t, u, v)$, i.e.

$$
R_{2}(t, x, u, v)=\sum_{p+q+\alpha \geq 2} a_{p, q, \alpha}(x) t^{p} u^{q} v^{\alpha} .
$$


We let $R>0$ small enough, such that

(i) $\frac{a_{p, q, \alpha}(x)}{c(x)}$ is holomorphic on $D_{R}=\{x \in \mathbf{C}|| x \mid \leq R\}$;

(ii) $\left|\frac{a_{p, q, \alpha}(x)}{c(x)}\right| \leq A_{p, q, \alpha}$ on $D_{R}$;

(iii) $\sum_{p+q+\alpha>2} A_{p, q, \alpha} t^{p} Y^{q} Y^{\alpha}$ is a convergent power series in $(t, Y)$.

Secondly from the equation (4), we have

$$
\begin{aligned}
&\left(x \frac{\partial}{\partial x}+\lambda_{1}\right) u_{1}=-\frac{\alpha(x)}{c(x)}, \\
& \vdots \\
&\left(x \frac{\partial}{\partial x}+\lambda_{k}\right) u_{k}=-\sum_{p+q+\alpha \geq 2} \sum_{\substack{p+k_{1}+\cdots+k_{q} \\
+l_{1}+\cdots+l_{\alpha}=k}} \frac{a_{p, q, \alpha}(x)}{c(x)} \times u_{k_{1}} \times \cdots \times u_{k_{q}} \\
& \quad \times \frac{\partial u_{l_{1}}}{\partial x} \times \cdots \times \frac{\partial u_{l_{\alpha}}}{\partial x}
\end{aligned}
$$

Without loss of generality, we may assume that the estimate (6) in Lemma 1 holds for any $k \geq 1$, i.e.

$$
\left\|u_{k}\right\|_{r} \leq \frac{C}{k}\left\|\tilde{f}_{k-1}\right\|_{r}, \text { for } k \geq 1,
$$

where $C$ is independent of $r$. Then we choose $A>0$, suth that on $D_{R}$,

$$
\left|u_{1}(x)\right| \leq A \text { and } \quad\left|\frac{\partial u_{1}}{\partial x}\right| \leq A .
$$

Now we introduce a function $Y(t)$, satisfying the following equation:

$$
Y=A t+\frac{C}{R-r} \sum_{p+q+\alpha \geq 2} \frac{A_{p, q, \alpha}}{(R-r)^{p+q+\alpha-2}} t^{p} Y^{q}(e Y)^{\alpha},
$$

where $r$ is a parameter with $0<r<R$.

Since the equation (11) is an analytic functional equation in $Y$, then we can use the implicit function theorem to deduce that the equation (11) has a unique holomorphic solution $Y(t)$ in a neighborhood of $t=0$ with $Y(0)=0$.

Expanding $Y(t)$ into Taylor series in $t$,

$$
Y(t)=\sum_{k \geq 1} Y_{k} t^{k}
$$


From the equation (11), we know that the coefficients of (12) can be given by

$$
Y_{1}=A
$$

and for $k \geq 2$

$$
\begin{aligned}
Y_{k}=\frac{C}{R-r} \sum_{p+q+\alpha \geq 2} \sum_{\substack{p+k_{1}+\ldots+k_{q} \\
+l_{1}+\cdots+l_{\alpha}=k}} \frac{A_{p, q, \alpha}}{(R-r)^{p+q+\alpha-2}} & \times Y_{k_{1}} \times \cdots \times Y_{k_{q}} \\
& \times\left(e Y_{l_{1}}\right) \times \cdots \times\left(e Y_{l_{\alpha}}\right) .
\end{aligned}
$$

Moreover we can deduce that $Y_{k}$ is of the form

$$
Y_{k}=\frac{C_{k}}{(R-r)^{k-1}}, \text { for } k=1,2, \cdots
$$

where $C_{1}=A$, and the constants $C_{k}>0$, for $k \geq 2$, can be decided inductively from the equation (13), which are independent of $r$. Actually from (13), it is easy to check that the order of $\frac{1}{(R-r)}$ is $k-1$, i.e. $1+(p+q+$ $\alpha-2)+\left(k_{1}-1\right)+\cdots+\left(k_{q}-1\right)+\left(l_{1}-1\right)+\cdots+\left(l_{\alpha}-1\right)=k-1$, so the formula (14) will be hold.

Next, we prove that the series $\sum_{k>1} Y_{k} t^{k}$ is a majorant series for the formal series solution $\sum_{k \geq 1} u_{k}(x) t^{k}$ near $x=0$. In fact, we can prove, by induction, that for any $k \geq 1$ and $0<r<R$, we have

$$
\begin{gathered}
\left|u_{k}(x)\right| \leq\left|k u_{k}(x)\right| \leq Y_{k}, \text { on } D_{r} ; \\
\left|\frac{\partial u_{k}}{\partial x}(x)\right| \leq e Y_{k}, \text { on } D_{r} .
\end{gathered}
$$

Actually, since $Y_{1}=A$, the estimates $(I)$ and $(I I)$ hold for $k=1$. Suppose that $k \geq 2$, and for any $1 \leq i<k,(I)$ and $(I I)$ are all hold for $i$. Then for $i=k$, from the equation (10) and Lemma 1 (here the estimate (6) holds for any $k \geq 1$ ) and inductive assumptions, we have

$$
\begin{aligned}
\left|u_{k}(x)\right| \leq \frac{C}{k} \sum_{p+q+\alpha \geq 2} \sum_{\substack{p+k_{1}+\ldots+k_{q} \\
+l_{1}+\cdots+l_{\alpha}=k}} A_{p, q, \alpha} \times\left|u_{k_{1}}(x)\right| & \times \cdots \times\left|u_{k_{q}}(x)\right| \\
& \times\left|\frac{\partial u_{l_{1}}}{\partial x}(x)\right| \times \cdots \times\left|\frac{\partial u_{l_{\alpha}}}{\partial x}(x)\right| \\
\leq \frac{C}{k} \sum_{p+q+\alpha \geq 2} \sum_{\substack{p+k_{1}+\ldots+k_{q} \\
+l_{1}+\cdots+l_{\alpha}=k}} A_{p, q, \alpha} \times Y_{k_{1}} & \times \cdots \times Y_{k_{q}} \\
& \times\left(e Y_{l_{1}}\right) \times \cdots \times\left(e Y_{l_{\alpha}}\right) .
\end{aligned}
$$


Since $R$ is small enough, we may assume $0<R<1$, thus $(R-r)^{p+q+\alpha-2}<$ 1 , then we have

$$
\begin{aligned}
\left|u_{k}(x)\right| \leq \frac{C}{k} \sum_{p+q+\alpha \geq 2} \sum_{\substack{p+k_{1}+\ldots+k_{q} \\
+l_{1}+\cdots+l_{\alpha}=k}} \frac{A_{p, q, \alpha}}{(R-r)^{p+q+\alpha-2}} & \times Y_{k_{1}} \times \cdots \times Y_{k_{q}} \\
& \times\left(e Y_{l_{1}}\right) \times \cdots \times\left(e Y_{l_{\alpha}}\right) .
\end{aligned}
$$

From the formule (13) and (14), we have

$$
\left|u_{k}(x)\right| \leq \frac{R-r}{k} Y_{k}=\frac{C_{k}}{k} \cdot \frac{1}{(R-r)^{k-2}} .
$$

Thus

$$
\left|u_{k}(x)\right| \leq\left|k u_{k}(x)\right| \leq \frac{C_{k}}{(R-r)^{k-2}} \leq \frac{C_{k}}{(R-r)^{k-1}}=Y_{k},
$$

the estimate $(I)$ holds.

Next, by using Lemma 2, we have

$$
\left|\frac{\partial u_{k}}{\partial x}(x)\right| \leq \frac{k-1}{k} \cdot \frac{e C_{k}}{(R-r)^{k-1}} \leq e Y_{k},
$$

this implies the estimate $(I I)$. Therefore we have proved that $\sum_{k \geq 1} Y_{k} t^{k}$ is the majorant series of the formal series solution (3) near $x=0$, thus the formal series solution (3) is convergent near $(0,0) \in \mathbf{C}_{t} \times \mathbf{C}_{x}$, Theorem 1 is proved.

\section{Case of High Order Singular PDE}

In this section, we shall extend the result of Theorem 1 to the case of high order singular partial differential equations. Let us consider the following high order singular partial differential equation:

$$
\left(t \partial_{t}\right)^{m} u=F\left(t, x,\left\{\left(t \partial_{t}\right)^{j} \partial_{x}^{\alpha} u\right\} \underset{\substack{j+\alpha \leq m \\ j<m}}{j \leq m}\right), \quad(t, x) \in \mathbf{C}_{t} \times \mathbf{C}_{x} .
$$

Now we denote $\left(t \partial_{t}\right)^{j} \partial_{x}^{\alpha} u$ by notation $Z_{j, \alpha}$, i.e.

$$
\left(t \partial_{t}\right)^{j} \partial_{x}^{\alpha} u \leftrightarrow Z_{j, \alpha}, \quad \text { and }\left\{\left(t \partial_{t}\right)^{j} \partial_{x}^{\alpha} u\right\}_{\substack{j+\alpha \leq m \\ j<m}}^{\substack{j<m \\ j<m}} \rightarrow Z=\left\{Z_{j, \alpha}\right\}_{\substack{j+\alpha \leq m \\ j<m}}
$$


For the function $F(t, x, Z)$, we suppose

(H1)' $F(t, x, Z)$ is a holomorphic function defined in a neighborhood of the origin $(0,0,0) \in \mathbf{C}_{t} \times \mathbf{C}_{x} \times \mathbf{C}^{N}$, where $N=\#\{(j, \alpha) \mid j+\alpha \leq m, j<m\}$.

(H2)' $F(0, x, 0) \equiv 0$ near $x=0$.

Thus we rewrite $F(t, x, Z)$ near the origin as

$$
F(t, x, Z)=\frac{\partial F}{\partial t}(0, x, 0) t+\sum_{\substack{j+\alpha \leq m \\ j<m}} \frac{\partial F}{\partial Z_{j, \alpha}}(0, x, 0) Z_{j, \alpha}+R_{2}(t, x, Z),
$$

where the degree of $R_{2}(t, x, Z)$ with respect to $(t, Z)$ is greater than or equal to 2.

If $\frac{\partial F}{\partial Z_{j, \alpha}}(0, x, 0) \equiv 0$ near $x=0$ for any $\alpha>0$, then the equation (15) is called non-linear Fuchsian type partial differential equation. In this case the existence and uniqueness of holomorphic solution of (15) has been proved by Gérard and Tahara [3].

Here we shall consider the case for the equation (15) to be totally characteristic type. Denote $\frac{\partial F}{\partial t}(0, x, 0)$ by $a(x)$, and $\frac{\partial F}{\partial Z_{j, \alpha}}(0, x, 0)$ by $b_{j, \alpha}(x)$, then the equation (15) becomes

$$
c\left(x, t \partial_{t}, \partial_{x}\right) u=a(x) t+R_{2}(t, x, Z),
$$

where the operator

$$
c\left(x, t \partial_{t}, \partial_{x}\right)=\left(t \partial_{t}\right)^{m}-\sum_{\substack{j+\alpha \leq m \\ j<m}} b_{j, \alpha}(x)\left(t \partial_{t}\right)^{j} \partial_{x}^{\alpha}
$$

is a linear singular partial differential operator, its symbol is given by

$$
c(x, \rho, \xi)=\rho^{m}-\sum_{\substack{j+\alpha \leq m \\ j<m}} b_{j, \alpha}(x) \rho^{j} \xi^{\alpha} .
$$

We define the indicial operator of (15) as

$$
c\left(x, \lambda, \partial_{x}\right)=\left.\left[t^{-\lambda} c\left(x, t \partial_{t} . \partial_{x}\right) t^{\lambda}\right]\right|_{t=0}, \quad \text { for } \lambda \in \mathbf{C} .
$$

Denoting $c_{m}(x, \rho, \xi)$ as principal symbol of $c\left(x, t \partial_{t}, \partial_{x}\right)$, i.e.

$$
c_{m}(x, \rho, \xi)=\rho^{m}-\sum_{\substack{j+\alpha=m \\ j<m}} b_{j, \alpha}(x) \rho^{j} \xi^{\alpha} .
$$


Thus we suppose the following condition:

(H3)' $b_{j, \alpha}(x)=x^{\alpha} \tilde{b}_{j, \alpha}(x)$ for $j+\alpha \leq m$ and $j<m, \tilde{b}_{j, \alpha}(x)$ are holomorphic near $x=0$ and $\tilde{b}_{0, m}(0) \neq 0$

Under the condition (H3)', the equation (15) is called totally characteristic type non-linear singular partial differential equation, and the operator $c\left(x, t \partial_{t}, \partial_{x}\right)$ is a linear partial differential operator with regular singularity at $(0,0) \in \mathbf{C}_{t} \times \mathbf{C}_{x}$. Furthermore, the principal symbol $c_{m}$ can be factorized as

$$
c_{m}(x, \rho, \xi)=\rho^{m}-\sum_{\substack{j+\alpha=m \\ j<m}} \tilde{b}_{j, \alpha}(x) \rho^{j}(x \xi)^{\alpha}=\tilde{b}_{0, m}(x) \prod_{j=1}^{m}\left(x \xi-\xi_{j}(x, \rho)\right),
$$

where $\xi_{j}(x, \rho)$ is a continuous function near $x=0$ and is homogeneous in $\rho$ of degree 1 , and here we suppose

(H4)' For every $j, 1 \leq j \leq m, \operatorname{Re} \xi_{j}(0,1)<0$.

For any $k \geq 1$, operator $c\left(x, k, \partial_{x}\right)$ is a Fuchsian operator in $x$, its indicial polynomial is defined as

$$
L(k, \lambda)=\left.\left[x^{-\lambda} c\left(x, k, \partial_{x}\right) x^{\lambda}\right]\right|_{x=0},
$$

thus roots of the indicial polynomial are called indicial exponents of $c\left(x, k, \partial_{x}\right)$. We have following result:

Theorem 2. Under the conditions (H1)', (H2)', (H3)' and (H4)', if for any $k \geq 1 L(k, \lambda) \neq 0$ for any $\lambda \in \mathbf{Z}_{+}=\{0,1,2, \cdots\}$, then the equation (15) has a unique holomorphic solution $u(t, x)$ near $(0,0) \in \mathbf{C}_{t} \times \mathbf{C}_{x}$ with $u(0, x) \equiv 0$ near $x=0$.

In order to prove Theorem 2, we let

$$
u(t, x)=\sum_{k=1}^{\infty} u_{k}(x) t^{k}
$$

be the formal series solution of the equation (15), then we introduce this formal series into the equation (17), and comparing the coefficients of $t^{k}$, for 
$k \geq 1$, in both sides of the equation, we obtain

$$
\begin{aligned}
c\left(x, 1, \partial_{x}\right) u_{1}(x) & =a(x), \\
& \vdots \\
c\left(x, k, \partial_{x}\right) u_{k}(x) & =f_{k-1}\left(\left\{\partial_{x}^{\alpha} u_{i} ; 1 \leq i \leq k-1,0 \leq \alpha \leq m\right\}\right),
\end{aligned}
$$

where $f_{k-1}$ is a holomorphic function near $x=0$, only depending on $\left\{\partial_{x}^{\alpha} u_{i} ; 1 \leq\right.$ $i \leq k-1,0 \leq \alpha \leq m\}$.

Since $L(k, \lambda) \neq 0$, for any $k \geq 1$ and $\lambda \in \mathbf{Z}_{+}$, we can solve the functions $u_{k}(x)$ uniquely from the equation (21), which are holomorphic in a common neighborhood of $x=0$. It remains to prove the convergence of the formal seires solution $(20)$.

Similar to Lemma 1, for high order equation we also have the following lemma, which is important in the proof of Theorem 2.

Lemma 3. There exists a constant $C>0$, suth that for $k$ large enough, we have

$$
\left\|u_{k}\right\|_{r} \leq \frac{C}{k^{m}}\left\|f_{k-1}\right\|_{r}
$$

where $r>0$ small enough.

Proof: First, similar to (19), we can also use the condition (H3)' and factorize the operator $c\left(x, k, \partial_{x}\right)$ into

$$
c(x, k, \xi)=\tilde{b}_{0, m}(x) \prod_{j=1}^{m}\left(x \xi-\tilde{\xi}_{j}(x, k)\right), \quad \tilde{b}_{0 . m}(0) \neq 0 .
$$

Thus near $x=0$, the equation (21) becomes

$$
\prod_{j=1}^{m}\left(x \partial_{x}-\tilde{\xi}_{j}(x, k)\right) u_{k}(x)=\tilde{f}_{k-1}
$$

where $\tilde{f}_{k-1}=\tilde{b}_{0, m}^{-1}(x) f_{k-1}$, and we know that $\xi_{j}(x, k)$ in the formula (19) is the main part of $\tilde{\xi}_{j}(x, k)$, which means

$$
\frac{\tilde{\xi}_{j}(x, k)}{\xi_{j}(x, k)} \rightarrow 1, \text { as } k \rightarrow \infty .
$$


We set

$$
V_{1}=\prod_{j=2}^{m}\left(x \partial_{x}-\tilde{\xi}_{j}(x, k)\right) u_{k},
$$

then the equation (23) becomes

$$
\left(x \partial_{x}-\tilde{\xi}_{1}(x, k)\right) V_{1}=\tilde{f}_{k-1} .
$$

Thus we have

$$
V_{1}=\int_{0}^{x} e^{\int_{y}^{x} \frac{\tilde{\xi}_{1}(s, k)}{s} d s} \frac{\tilde{f}_{k-1}(y)}{y} d y .
$$

Since $\xi_{j}(x, k)$ is homogenous of degree 1 with respect to $k$, from (24) we have

$$
\operatorname{Re} \tilde{\xi}_{j}(0, k)=k \operatorname{Re} \xi_{j}(0,1)+o(k), \quad \text { for } 1 \leq j \leq m .
$$

Thus from the condition (H4)' we have $\operatorname{Re} \tilde{\xi}_{j}(0, k)<0(1 \leq j \leq m)$ for $k$ large enough. So, similar to the proof of Lemma 1, near the origin and for large $k$ we have for some positive constants $c$ and $C_{1}$

$$
\left|e^{\int_{y}^{x} \frac{\tilde{\xi}_{1}(s, k)}{s} d s}\right| \leq c e^{C_{1} \operatorname{Re} \tilde{\xi}_{1}(0, k) \ln \left(\frac{|x|}{|y|}\right)} \mid=c\left(\frac{|x|}{|y|}\right)^{C_{1} \operatorname{Re} \tilde{\xi}_{1}(0, k)},
$$

that is for large $k,-C_{1} \tilde{\xi}_{1}(0, k)>0$ and we have

$$
\begin{aligned}
\left\|V_{1}\right\|_{r} & \leq c \max _{|x| \leq r}\left(|x|^{C_{1} \operatorname{Re} \tilde{\xi}_{1}(0, k)} \int_{0}^{|x|} \eta^{-C_{1} \operatorname{Re} \tilde{\xi}_{1}(0, k)-1} d \eta\right)\left\|\tilde{f}_{k-1}\right\|_{r} \\
& \leq \frac{c}{-C_{1} \operatorname{Re} \tilde{\xi}_{1}(0, k)}\left\|\tilde{f}_{k-1}\right\|_{r} .
\end{aligned}
$$

Next, let $V_{2}=\prod_{j=3}^{m}\left(x \partial_{x}-\tilde{\xi}_{j}(x, k)\right) u_{k}$, then $\left(x \partial_{x}-\tilde{\xi}_{2}(x, k)\right) V_{2}=V_{1}$. It is the same we can deduce that there exists a constant $C_{2}>0$, such that for large $k$

$$
\left\|V_{2}\right\|_{r} \leq \frac{c}{-C_{2} \operatorname{Re} \tilde{\xi}_{2}(0, k)}\left\|V_{1}\right\|_{r} \leq \frac{c^{2}}{C_{1} C_{2} \operatorname{Re} \tilde{\xi}_{1}(0, k) \operatorname{Re} \tilde{\xi}_{2}(0, k)}\left\|\tilde{f}_{k-1}\right\|_{r} .
$$

So finally we have for $k$ large enough,

$$
\left\|u_{k}\right\|_{r} \leq \prod_{j=1}^{m}\left(\frac{c}{-C_{j} \operatorname{Re} \tilde{\xi}_{j}(0, k)}\right)\left\|\tilde{f}_{k-1}\right\|_{r}, \quad\left(\exists C_{j}>0\right) .
$$


From (25), we know that there exists a constant $C^{\prime}>0$, such that for $k$ large enough we have

$$
\left\|u_{k}\right\|_{r} \leq \frac{C^{\prime} c^{m}}{k^{m}}\left\|\tilde{f}_{k-1}\right\|_{r} \leq \frac{c_{1} C^{\prime} c^{m}}{k^{m}}\left\|f_{k-1}\right\|_{r}
$$

where

$$
\max _{|x| \leq r}\left|\tilde{b}_{0, m}^{-1}(x)\right| \leq c_{1},
$$

for $r>0$ small enough. Lemma 3 is proved.

By using Lemma 3, we can prove the convergence of the formal series solution (20) in the following way.

First we expand the remainder term $R_{2}(t, x, Z)$ of (16) into Taylor series with respect to $(t, Z)$, i.e.

$$
R_{2}(t, x, Z)=\sum_{p+|\nu| \geq 2} a_{p, \nu}(x) t^{p} Z^{\nu}
$$

where

$$
\nu=\left\{\nu_{j, \alpha}\right\}_{\substack{j+\alpha \leq m \\ j<m}} \in \mathbb{N}^{N}, \quad|\nu|=\sum_{\substack{j+\alpha \leq m \\ j<m}} \nu_{j, \alpha}, \quad Z^{\nu}=\prod_{\substack{j+\alpha \leq m \\ j<m}}\left(Z_{j, \alpha}\right)^{\nu_{j, \alpha}} .
$$

We let $R>0$ small enough such that $0<R<1$ and

(i) $a_{p, \nu}(x)$ is holomorphic on $D_{R}$;

(ii) $\left|a_{p, \nu}(x)\right| \leq A_{p, \nu}$ on $D_{R}$;

(iii) $\sum_{p+|\nu| \geq 2} A_{p, \nu} t^{p} Z^{\nu}$ is a convergent power series in $(t, Z)$.

For simplicity we assume that the estimate (22) holds for any $k \geq 1$. Let us choose $A>0$ so that

$$
\left|\partial_{x}^{\alpha} u_{1}(x)\right| \leq A \quad \text { on } D_{R}, \quad 0 \leq \forall \alpha \leq m .
$$

Now, let us consider the following functional equation:

$$
Y=A t+\frac{C}{(R-r)^{m}} \sum_{p+|\nu| \geq 2} \frac{A_{p, \nu}}{(R-r)^{m(p+|\nu|-2)}} t^{p}(B Y)^{|\nu|},
$$

where $r$ is a parameter with $0<r<R, C>0$ is the constant in (22), and $B=(m e)^{m}$. 
Since the equation (27) is an analytic functional equation in $\mathrm{Y}$, we see by the implicit function theorem that the equation (27) has a unique holomorphic solution $Y(t)$ in a neighborhood of $t=0$ with $Y(0)=0$.

If we expand $Y(t)$ into

$$
Y=\sum_{k \geq 1} Y_{k} t^{k}
$$

then by the same argument as in the proof of Theorem 1 (and also in the discussion in chapter 6 of [4]) we can show that for any $k \geq 1$ and $0<r<R$ we have

$$
\begin{gathered}
\quad\left|k^{j} \partial_{x}^{\alpha} u_{k}(x)\right| \leq(m e)^{\alpha} Y_{k} \leq B Y_{k} \text { on } D_{r} \\
\text { for any }(j, \alpha) \text { with } j+\alpha \leq m \text { and } j<m \text {. }
\end{gathered}
$$

This implies that $Y=\sum_{k>1} Y_{k} t^{k}$ is a majorant series of the formal solution (20). Thus, we have obtained the convergence of the formal series solution (20). Theorem 2 is proved.

Acknowledgement The work was supported by the NNSFC and Sophia Lecturing-Research Grants of Sophia University, Tokyo.

The research was started while CH was a visiting professor in Math. Dept., Sophia University of Tokyo during April - July 1997. Also the final version of the paper was done while CH was a visiting professor in Math. Institute, Potsdam University of Germany during July 1998. CH would like to thank these Institutes for the invitations and their hospitality 


\section{References}

[1] R. Gérard and H. Tahara : Nonlinear singular first order partial differential equations of Briot-Bouquet type, Proc. Japan Acad., 66 (1990), $72-74$.

[2] R. Gérard and H. Tahara : Holomorphic and singular solutions of nonlinear singular first order partial differential equations, Publ. RIMS, Kyoto Univ. 26 (1990), 979-1000.

[3] R. Gérard and H. Tahara : Solutions holomorphes et singulières d'équations aux dérivées partielles singulières non linéaires, Publ. RIMS, Kyoto Univ. 29 (1993), 121-151.

[4] R. Gérard and H. Tahara : Singular nonlinear partial differential equations, Aspects of Mathematics, E 28, Vieweg, 1996

[5] E. Hille: Ordinary differential equations in the complex domain, John Wiley and Sons, 1976.

[6] L. Hörmander : Linear partial differential operators, Springer, 1963.

[7] H. Yamane: Nonlinear singular first order partial differential equations whose characteristic exponent takes a positive integer value, Publ. RIMS, Kyoto Univ., 33 (1997), 801-811.

CHEN Hua:

Department of Mathematics, Wuhan University, Wuhan, 430072, China

Email: chenhua@whu.edu.cn

TAHARA Hidetoshi:

Department of Mathematics, Sophia University, Tokyo, 102, Japan

Email: h-tahara@hoffman.cc.sophia.ac.jp 